\title{
Across the Finnish line
}

Data from the Finnish arm of the European Randomized Study of Screening for Prostate Cancer has indicated that three serum-PSA-based screening rounds are required in order to reduced subsequent prostate cancer incidence.

Pakarainen and colleagues separated 29,298 men who participated in the Finnish section of the European study into subgroups depending on whether they had taken part in one, two or three screening rounds. They formed a reference group of 43,151 age-matched men from the control arm of the trial and new prostate cancer cases diagnosed after screening were identified from the Finnish Cancer Registry.

The cumulative incidence of prostate cancer diagnosed in screened men after their last screening attendance was $5.2 \%$, and incidence in nonscreened men was $6.2 \%$. Prostate cancer incidence in men screened once was 11.2 cases $/ 1,000$ person years compared with 8.1 cases for men in the control group; for men screened twice, incidence was 8.9 cases $/ 1,000$ person years versus 9.1 cases $/ 1,000$ person years for nonscreened men; for men screened three times, incidence was 4.5 cases $/ 1,000$ person years versus 7.9 cases $/ 1,000$ person years for men in the control arm. In analysis of high-grade cancers, reduction of prostate cancer incidence was only observed for men who attended two or three screening rounds.

These results indicate that repeated screening cycles are needed in order to reduce postscreening prostate cancer incidence, with a minimum of two rounds required to decrease the incidence of high-grade cancer.

Louise Stone

ORIGINAL ARTICLE Pakarainen, T. et al. Number of screening rounds and postscreening prostate cancer incidence: results from the Finnish section of the European Randomized Study of Screening for Prostate Cancer study. Eur. Urol. http://dx.doi. org/10.1016/j.eururo.2016.05.009 (2016) 